\title{
COMMON LOCAL SPECTRAL PROPERTIES OF INTERTWINING LINEAR OPERATORS
}

\author{
Jong-KWANG YoO AND HyUk HaN
}

\begin{abstract}
Let $T \in \mathcal{L}(X), S \in \mathcal{L}(Y), A \in \mathcal{L}(X, Y)$ and $B \in$ $\mathcal{L}(Y, X)$ such that $S A=A T, T B=B S, A B=S$ and $B A=T$. Then $S$ and $T$ shares that same local spectral properties SVEP, property $(\beta)$, property $(\beta)_{\epsilon}$, property $(\delta)$ and decomposability. From these common local spectral properties, we give some results related with Aluthge transforms and subscalar operators.
\end{abstract}

\section{Introduction}

Let $X$ and $Y$ be Banach spaces over the complex plane $\mathbb{C}$, let $\mathcal{L}(X, Y)$ be the space of all bounded linear operators from $X$ to $Y$. And let $\mathcal{L}(X)$ denote the Banach algebra of all bounded linear operators on a Banach space $X$.

Given an operator $T \in \mathcal{L}(X), \sigma(T)$ denotes the spectrum of $T$ and Lat $(T)$ denotes the collection of all closed $T$-invariant linear subspaces of $X$, and for an $Y \in \operatorname{Lat}(T), T \mid Y$ denotes the restriction of $T$ on $Y$. An operator $T \in \mathcal{L}(X)$ is called decomposable if for every open covering $\{U, V\}$ of the complex plane $\mathbb{C}$, there exist $Y, Z \in \operatorname{Lat}(T)$ such that

$$
\sigma(T \mid Y) \subset U, \sigma(T \mid Z) \subset V \text { and } Y+Z=X .
$$

It has been shown by Albrecht[1] that this simple definition of operator decomposability is equivalent to the original definition due to Foias[5]. Decomposable operators are rich. For example, normal operators, spectral operators in the sense of Dunford, operators with totally disconnected spectrum and hence compact operators are decomposable[7].

Received March 30, 2009. Accepted May 19, 2009.

2000 Mathematics Subject Classification: 47A11, 47A53.

Key words and phrases: Local Spectral Theory, Bishop's Property $\beta$, Decomposable Operators, Subscalar Operators.

Corresponding author : Hyuk Han(hyukhan@kongju.ac.kr). 
Let $D(\lambda, r)$ be the open disc centered at $\lambda \in \mathbb{C}$ with radius $r>0$. We say that $T$ has the single valued extension property, abbreviate it SVEP, at $\lambda \in \mathbb{C}$ if there exists $r>0$ such that for every open subset $U \subset D(\lambda, r)$, the only analytic solution of the equation $(T-\mu) f(\mu)=0$ is the constant function $f \equiv 0$. We define the analytic residuum, denoted by $\mathcal{S}(T)$, the open set where $T$ fails to have the constant function $f \equiv 0$. An operator $T \in \mathcal{L}(X)$ said to have the single-valued extension property, when $T$ satisfies this property at every complex number. Hence $T$ has the SVEP if and only if $\mathcal{S}(T)=\emptyset$.

Given an arbitrary operator $T \in \mathcal{L}(X)$ and $x \in X$, the local resolvent set $\rho_{T}(x)$ of $T$ at $x \in X$ is defined as the set of all $\lambda \in \mathbb{C}$ for which there exist an analytic $X$-valued function $f$ on some open neighborhood $U$ of $\lambda$ such that $(T-\mu) f(\mu)=x$ for all $\mu \in U$. The complement of the local resolvent set is said to be the local spectrum and denoted by $\sigma_{T}(x)$. That is,

$$
\sigma_{T}(x)=\mathbb{C} \backslash \rho_{T}(x) .
$$

It may happen that the local spectrum $\sigma_{T}(x)$ is the empty set.

Let $U$ be an open subset of the complex plane and $\mathcal{O}(U, X)$ be the Fréchet algebra of all analytic $X$-valued functions on $U$ endowed with uniform convergence on compact sets of $U$. The operator $T$ is said to satisfy Bishop's property $(\beta)$ at $\lambda \in \mathbb{C}$ if there exists $r>0$ such that for every open subset $U \subset D(\lambda, r)$ and for any sequence $\left\{f_{n}\right\} \subset \mathcal{O}(U, X)$, if $\lim _{n \rightarrow \infty}(T-\mu) f_{n}(\mu)=0$ in $\mathcal{O}(U, X)$, then $\lim _{n \rightarrow \infty} f_{n}(\mu)=0$ in $\mathcal{O}(U, X)$. We denote by $\sigma_{\beta}(T)$ by the set where $T$ fails to satisfy $(\beta)$ and we say that $T$ satisfies Bishop's property $(\beta)$ precisely when $\sigma_{\beta}(T)=\emptyset$.

An operator $T \in L(X)$ is said to have the decompositions property $(\delta)$ if given an arbitrary open covering $\{U, V\}$ of $\mathbb{C}$ and for every $x \in X$ there exist a pair of elements $u, v \in X$ and a pair of analytic functions $f: \mathbb{C} \backslash U^{-} \longrightarrow X$ and $g: \mathbb{C} \backslash V^{-} \longrightarrow X$ such that $x=u+v$,

$$
\begin{array}{lll}
u=(T-\lambda) f(\lambda) & \text { for all } & \lambda \in \mathbb{C} \backslash U^{-}, \\
v=(T-\lambda) g(\lambda) & \text { for all } & \lambda \in \mathbb{C} \backslash V^{-} .
\end{array}
$$

It is well known that the properties $(\beta)$ and $(\delta)$ are dual to each other[7]. That is, the operator $T$ satisfies has Bishop's property $(\beta)$ if and only if its adjoint $T^{*}$ satisfies the decomposition property $(\delta)$ on the dual space, and if two properties are interchanged the corresponding statement true. Also it is well known that $T$ is decomposable in the sense of Foias if and only if $T$ satisfies both $(\beta)$ and $(\delta)$, and hence $T$ is decomposable if and only if $T^{*}$ is decomposable. It has also been shown that an operator $T \in L(X)$ has property $(\beta)$ if and only if $T$ is similar to 
the restriction of a decomposable operator to one of its closed invariant subspaces and an operator $T \in L(X)$ has property $(\delta)$ if and only if $T$ is similar to a quotient of a decomposable operator[7].

The property $(\beta)_{\epsilon}$ is defined in a similar way as for property $(\beta)$. To be precise; let $\mathcal{E}(U, X)$ be the Fréchet algebra of all infinitely differentiable $X$-valued functions on $U \subset \mathbb{C}$ endowed with the topology of uniform convergence on compact subsets of $U$ of all derivatives. The operator $T \in \mathcal{L}(X)$ is said to have property $(\beta)_{\epsilon}$ at $\lambda \in \mathbb{C}$ if there exists $U$ a neighborhood of $\lambda$ such that for each open set $O \subset U$ and for any sequence $\left\{f_{n}\right\}$ of $X$-valued functions in $\mathcal{E}(O, X)$ the convergence of $(T-\mu) f_{n}(\mu)$ to zero in $\mathcal{E}(O, X)$ yields to the convergence of $f_{n}$ to zero in $\mathcal{E}(O, X)$. Denote by $\sigma_{(\beta)_{\epsilon}}(T)$ the set where $T$ fails to satisfy $(\beta)_{\epsilon}$. We will say that $T$ satisfies property $(\beta)_{\epsilon}$ if $\sigma_{(\beta)_{\epsilon}}(T)=\emptyset$.

An important generalization of normal operators to the setting of Banach spaces is the class of generalized scalar operators. We denote by $C^{\infty}(\mathbb{C})$ the Fréchet algebra of all infinitely differentiable complex valued functions $\varphi(z), z=x_{1}+i x_{2}, x_{1}, x_{2} \in \mathbb{R}$, defined on the complex plane $\mathbb{C}$ with the topology of uniform convergence of every derivative on each compact subset of $\mathbb{C}$. That is, with the topology generated by the family of pseudo-norm

$$
|\varphi|_{K, m}=\max _{|p| \leq m} \sup _{z \in K}\left|D^{p} \varphi(z)\right|,
$$

where $K$ is an arbitrary compact subset of $\mathbb{C}, m$ a non-negative integer, $p=\left(p_{1}, p_{2}\right), p_{1}, p_{2} \in \mathbb{N},|p|=p_{1}+p_{2}$ and

$$
D^{p} \varphi=\frac{\partial^{|p|} \varphi}{\partial x_{1}^{p_{1}} \partial x_{2} p_{2}}, \quad\left(z=x_{1}+i x_{2}\right) .
$$

An operator $T \in L(X)$ on a complex Banach space $X$ is called a generalized scalar operator if there exists a continuous algebra homomorphism $\Phi: C^{\infty}(\mathbb{C}) \rightarrow L(X)$ satisfying $\Phi(1)=I$ and $\Phi(z)=T$, where $I$ is the identity operator on $X$ and $z$ denotes the identity function on $\mathbb{C}$. Such a continuous function $\Phi$ is in fact an operator valued distribution and it is called a spectral distribution for $T$. The class of generalized scalar operators were introduced by Colojoară and Foiás[5]. An important subclass of the decomposable operators is formed by the generalized scalar operators. An operator $T \in \mathcal{L}(X)$ on a complex Banach space $X$ is said to be subscalar if $T$ is similar to the restriction of a generalized scalar operator to one of its closed invariant subspaces. It is clear that every subscalar operator has property $(\beta)$, since the restriction of an operator with property $(\beta)$ to a closed invariant subspace certainly inherits this 
property. Moreover it is well known that $T \in \mathcal{L}(X)$ is subscalar if and only if $T$ has property $(\beta)_{\epsilon}[6]$.

Let $H$ be a Hilbert space over the complex plane $\mathbb{C}$ with the inner product $\langle\cdot, \cdot\rangle$. An operator $T \in \mathcal{L}(H)$ is said to be hyponormal if its self commutator $\left[T^{*}, T\right]=T^{*} T-T T^{*}$ is positive, that is,

$$
\left\langle\left(T^{*} T-T T^{*}\right) \xi, \xi\right\rangle \geq 0,
$$

or equivalently, $\left\|T^{*} \xi\right\| \leq\|T \xi\|$ for every $\xi \in H$. It is well known that hyponormal operators on a Hilbert space $H$ is subscalar [9].

\section{Common local spectral properties of intertwining Linear Operators}

Theorem 1. Let $T \in \mathcal{L}(X), S \in \mathcal{L}(Y), A \in \mathcal{L}(X, Y)$ and $B \in$ $\mathcal{L}(Y, X)$ such that $S A=A T$ and $T B=B S$. Suppose that $A B=S$ and $B A=T$. Then $T$ has the single valued extension property (resp. property $(\beta))$ at $\lambda \in \mathbb{C}$ if and only if $S$ has the single valued extension property (resp. property $(\beta)$ ) at $\lambda \in \mathbb{C}$. Moreover, $T$ has the single valued extension property (resp. property $(\beta))$ if and only if $S$ has the single valued extension property (resp. property $(\beta)$ ).

Proof. We only give the proof for property $(\beta)$, the case of the single valued extension property is similar. Let $\lambda \in \mathbb{C} \backslash \sigma_{\beta}(S)$ and let $\left\{f_{n}\right\}$ be a sequence of $X$-valued analytic functions in a open neighborhood $U$ of $\lambda$ such that $\lim _{n \rightarrow \infty}(T-\mu) f_{n}(\mu)=0$ in $\mathcal{O}(U, X)$. Then we have,

$$
\begin{aligned}
0 & =\lim _{n \rightarrow \infty} A(T-\mu) f_{n}(\mu) \\
& =\lim _{n \rightarrow \infty}(S-\mu) A f_{n}(\mu)
\end{aligned}
$$

in $\mathcal{O}(U, Y)$. Since $\lambda \in \mathbb{C} \backslash \sigma_{\beta}(S)$, it follows that $\lim _{n \rightarrow \infty} A f_{n}(\mu)=0$ in $\mathcal{O}(U, Y)$. Then we have,

$$
\begin{aligned}
0 & =\lim _{n \rightarrow \infty} B A f_{n}(\mu) \\
& =\lim _{n \rightarrow \infty} T f_{n}(\mu)
\end{aligned}
$$

in $\mathcal{O}(U, X)$. Since $\mu f_{n}(\mu)=T f_{n}(\mu)-(T-\mu) f_{n}(\mu)$, we deduce that $\left\{\mu f_{n}(\mu)\right\}$ converges to 0 on compact sets of $U$. Since $f_{n}$ is analytic, the maximum modulus principle implies $\left\{f_{n}\right\}$ converges to 0 on compact sets of $U$. Thus $\lambda \in \mathbb{C} \backslash \sigma_{\beta}(T)$. The reverse implication is obtained by the symmetry. 
By passing to adjoint in Theorem 1, and by using the duality of property $(\beta)$ and $(\delta)$, we obtain

Corollary 2. Let $T \in \mathcal{L}(X), S \in \mathcal{L}(Y), A \in \mathcal{L}(X, Y)$ and $B \in$ $\mathcal{L}(Y, X)$ such that $S A=A T$ and $T B=B S$. Suppose that $A B=S$ and $B A=T$. Then $T$ has the decomposition property $(\delta)$ if and only if $S$ has the decomposition property $(\delta)$. Moreover, $T$ is decomposable if and only if $S$ is decomposable.

The following lemma is found in [8].

Lemma 3. Let $O$ be an open subset of $\mathbb{C}$ and $\left\{f_{n}\right\}$ be a sequence in $\mathcal{E}(O, X)$ such that $\left\{\mu f_{n}(\mu)\right\}$ converges to zero in $\mathcal{E}(O, X)$. Then $\left\{f_{n}\right\}$ converges to zero in $\mathcal{E}(O, X)$.

Theorem 4. Let $T \in \mathcal{L}(X), S \in \mathcal{L}(Y), A \in \mathcal{L}(X, Y)$ and $B \in$ $\mathcal{L}(Y, X)$ such that $S A=A T$ and $T B=B S$. Suppose that $A B=S$ and $B A=T$. Then $\sigma_{(\beta)_{\epsilon}}(T)=\sigma_{(\beta)_{\epsilon}}(S)$. In particular, $T$ is subscalar if and only if $S$ is subscalar.

Proof. Suppose that $\lambda \in \mathbb{C} \backslash \sigma_{(\beta)_{\epsilon}}(S)$. Then there exists a neighborhood $O$ of $\lambda$ such that $O \cap \sigma_{(\beta)_{\epsilon}}(S)=\phi$. If $\left\{f_{n}\right\}$ is any sequence in $\mathcal{E}(O, X)$ such that $(T-\mu) f_{n}(\mu)$ converges to zero in $\mathcal{E}(O, X)$, then

$$
\begin{aligned}
0 & =\lim _{n \rightarrow \infty} A(T-\mu) f_{n}(\mu) \\
& =\lim _{n \rightarrow \infty}(S-\mu) A f_{n}(\mu) .
\end{aligned}
$$

in $\mathcal{E}(O, Y)$. Since $\lambda \in \mathbb{C} \backslash \sigma_{(\beta)_{\epsilon}}(S)$, it follows that $\lim _{n \rightarrow \infty} A f_{n}(\mu)=0$ in $\mathcal{E}(O, Y)$. Then we have,

$$
\begin{aligned}
0 & =\lim _{n \rightarrow \infty} B A f_{n}(\mu) \\
& =\lim _{n \rightarrow \infty} T f_{n}(\mu) .
\end{aligned}
$$

Since $\mu f_{n}(\mu)=T f_{n}(\mu)-(T-\mu) f_{n}(\mu)$, we deduce that $\left\{\mu f_{n}(\mu)\right\}$ converges to 0 in $\mathcal{E}(O, X)$. By Lemma $3,\left\{f_{n}\right\}$ converges to zero in $\mathcal{E}(O, X)$. Hence $\lambda \in \mathbb{C} \backslash \sigma_{(\beta)_{\epsilon}}(T)$. The reverse implication is obtained by the the symmetry.

Corollary 5. Let $A: X \longrightarrow Y$ and $B: Y \longrightarrow X$ be bounded linear operators. Then $A B$ has property $(\beta)($ resp. $(\delta)$ or decomposable or 
subscalar) if and only if $B A$ has property $(\beta)($ resp. $(\delta)$ or decomposable or subscalar).

Proof. Let $S=A B$ and $T=B A$. Then we have,

$$
S A=A B A=A T \quad \text { and } \quad T B=B A B=B S .
$$

Hence by Theorem 1 and Theorem 4, we have this corollary.

Let $T \in \mathcal{L}(H)$ be a bounded operator on a Hilbert space $H$ and $U|T|$ be the polar decomposition of $T$, where $|T|=\left(T T^{*}\right)^{\frac{1}{2}}$ and $U$ is the appropriate partial isometry. The generalized Aluthge transform associated with $T$ and $s, t \geq 0$ is defined by

$$
T(s, t)=|T|^{s} U|T|^{t} .
$$

In the case $s=t=\frac{1}{2}$, the operator

$$
\widetilde{T}=|T|^{\frac{1}{2}} U|T|^{\frac{1}{2}}
$$

is called the Aluthge transform of $T$ and was first considered by Aluthge[2] to extend some inequalities related to hyponormality. Let $s \leq t, A=$ $|T|^{r}$ and $B=|T|^{s} U|T|^{t-r}$. Then we have,

$$
A B=T(s+r, t-r) \quad \text { and } \quad B A=T(s, t) .
$$

Therefore, $T(s, t)$ and $T(s+r, t-r)$ almost have the same local spectral properties. In particular, $T$ and $\widetilde{T}$ almost have the same local spectral properties.

Corollary 6. Let $T \in \mathcal{L}(H), s \geq 0$ and $0 \leq r \leq t$. Then $T(s, t)$ has the property $(\beta)$ (resp. $(\delta)$ or decomposable or subscalar) if and only if $T(s+r, t-r)$ has the property $(\beta)$ (resp. $(\delta)$ or decomposable or subscalar).

Theorem 7. Let $T \in \mathcal{L}(X), S \in \mathcal{L}(Y), A \in \mathcal{L}(X, Y)$ and $B \in$ $\mathcal{L}(Y, X)$ such that $S A=A T, T B=B S$. Suppose that $A B=S$ and $B A=T$. Then

(1) $\sigma_{S}(A x) \subset \sigma_{T}(x) \subset \sigma_{S}(A x) \cup\{0\}$ for every $x \in X$.

(resp. $\sigma_{T}(B y) \subset \sigma_{S}(y) \subset \sigma_{T}(B y) \cup\{0\}$ for every $y \in Y$.)

(2) In particular, if $A$ is injective then $\sigma_{T}(x)=\sigma_{S}(A x)$ for every $x \in X$. (resp. if $B$ is injective then $\sigma_{S}(y)=\sigma_{T}(B y)$ for every $y \in Y$.

Proof. (1) Let $\lambda \notin \sigma_{T}(x)$ and $x(\mu)$ be an $X$-valued analytic function on a neighborhood $O$ of $\lambda$ such that $(T-\mu) x(\mu)=x$ for every $\mu \in O$. 
Since $S A=A T$, we have

$$
\begin{aligned}
A x & =A(T-\mu) x(\mu) \\
& =(S-\mu) A x(\mu)
\end{aligned}
$$

for all $x \in O$. Since $A x(\cdot): O \longrightarrow Y$ is an $Y$-valued analytic function on a neighborhood $O$ of $\lambda$, we have

$$
\lambda \notin \sigma_{S}(A x) .
$$

To show the second inclusion, let $\lambda \notin \sigma_{S}(A x) \cup\{0\}$ and $y(\mu)$ be an $Y$-valued analytic function on an open neighborhood $O$ of $\lambda$ with $0 \notin O$ such that $(S-\mu) y(\mu)=A x$ for all $\mu \in O$. Since $B A=T$ and $T B=B S$, we have

$$
\begin{aligned}
T x & =B A x \\
& =B(S-\mu) y(\mu) \\
& =(T-\mu) B y(\mu) .
\end{aligned}
$$

Therefore, we have

$$
T(B y(\mu)-x)=\mu B y(\mu) .
$$

Define the $X$-valued analytic function $z(\cdot): O \longrightarrow X$ by

$$
z(\mu)=\frac{1}{\mu}(B y(\mu)-x) .
$$

Then it is easy to see that

$$
x=(T-\mu) z(\mu) \quad \text { for every } \quad \mu \in O,
$$

and hence $\lambda \notin \sigma_{T}(x)$.

(2) Since $\sigma_{T+\lambda I}(x)=\sigma_{T}(x)+\lambda$ for every $\lambda \in \mathbb{C}$ and every $x \in X$, it suffices to consider the case $\lambda=0$. Suppose that $0 \in \sigma_{S}(A x)$. Then, by (1) of this Theorem we have,

$$
\sigma_{T}(x)=\sigma_{S}(A x) \quad \text { for every } \quad x \in X .
$$

Suppose that $0 \notin \sigma_{S}(A x)$ and let $y(\mu)$ be an $Y$-valued analytic function on a neighborhood $U$ of 0 such that $(S-\mu) y(\mu)=A x$ for every $\mu \in U$. Since $0 \in U, A B y(0)=S y(0)=A x$. From the injectivity of $A$, it follows that $B y(0)=x$. Moreover, we have

$$
\begin{aligned}
\mu y(\mu) & =S y(\mu)-A x \\
& =A(B y(\mu)-x) .
\end{aligned}
$$


Therefore, we have

$$
y(\mu)=A\left[\frac{1}{\mu}(B y(\mu)-x)\right]
$$

for every $\mu \in U \backslash\{0\}$. Define the function $z(\cdot): U \longrightarrow X$ by

$$
z(\mu)= \begin{cases}\frac{1}{\mu}(B y(\mu)-x) & \text { if } \mu \neq 0 \\ B y^{\prime}(0) & \text { if } \mu=0 .\end{cases}
$$

Then clearly $z(\mu)$ is analytic on $U$ and it is easily see that $A[x-(T-$ $\mu) z(\mu)]=0$ for every $\mu \in U$. Since $A$ is injective, we have

$$
(T-\mu) z(\mu)=x
$$

for every $\mu \in U$. Hence $0 \notin \sigma_{T}(x)$.

Corollary 8. Let $T \in \mathcal{L}(X), S \in \mathcal{L}(Y), A \in \mathcal{L}(X, Y)$ and $B \in$ $\mathcal{L}(Y, X)$ such that $S A=A T, T B=B S$. Suppose that $A B=S$ and $B A=T$. Suppose that $A$ and $B$ are injective. Then $\sigma(T)=\sigma(S)$.

Proof. For an arbitrary operator $T \in \mathcal{L}(X)$, the following equality is well known

$$
\sigma(T)=\bigcup_{x \in X} \sigma_{T}(x) \bigcup \mathcal{S}(T)
$$

Suppose that $A$ and $B$ are injective. Then by Theorem 1 and Theorem 7 we have,

$$
\begin{aligned}
\sigma(T) & =\bigcup_{x \in X} \sigma_{T}(x) \bigcup \mathcal{S}(T) \\
& =\bigcup_{x \in X} \sigma_{S}(A x) \bigcup \mathcal{S}(S) \\
& \subset \bigcup_{y \in Y} \sigma_{S}(y) \bigcup \mathcal{S}(S) \\
& =\sigma(S) .
\end{aligned}
$$

The reverse inclusion is obtained by the symmetry.

\section{References}

[1] E. Albrecht, On decomposable operators, Integral Equations Operator Theory, 2(1979), 1-10.

[2] A. Aluthge, On p-hyponormal operators for $0<p<1$, Integral Equations Operator Theory, 13(1990), 307-315. 
[3] C. Benhida and E. H. Zerouali, Local spectral theory of linear operators $R S$ and $S R$, Integral Equations and Operator Theory, 54(1)(2006), 1-8.

[4] Lin Chen, Yan Zikun and Ruan Yingbin, Common operator properties of operators $R S$ and $S R$ and p-Hyponormal operators, Integral Equations Operator Theory, 43(2002), 313-325.

[5] I. Colojoarvă and C. Foiás, Theory of Generalized Spectral Operators, Gorden and Breach, New York, 1968.

[6] J. Eschmeier and M. Putinar, Bishop's condition $(\beta)$ and rich extensions of linear operators, Indiana Univ. Math. J., 37(1988), 325-348.

[7] K. B. Laursen and M. M. Neumann, An Introduction to Local Spectral Theory, London Mathematical Society Monographs New Series 20, Oxford Science Publications, Oxford, 2000.

[8] B. Malgrange, Ideals of Differentiable Functions, Oxford University Press, London, 1967.

[9] M. Putinar, Hyponormal operators are subscalar, J. Operator Theory, 12(1984), 385-395.

Jong-Kwang Yoo

Department of Liberal Arts and Science

Chodang University

Muan 534-701, Korea

E-mail: jkyoo@chodang.ac.kr

Hyuk Han

Department of Liberal Arts

Kongju National University

Yesan 340-702, Korea

E-mail: hyukhan@kongju.ac.kr 